\title{
Ciudades Cambiantes: spazi aperti urbani in evoluzione per nuove città
}

\author{
Jordi Bellmunt \\ B2B arquitectes, Barcellona, \\ admin@b2barq.com
}

\begin{abstract}
This article reflects on the drift of public space in the Mediterranean cities, on the new trends from Tactical Urbanism to urban interventions that rethink the public character of our cities. The city is a complex organism that cannot simplify itself at will, it has its inertia, its time and its difficulty in absorbing political ideologies. The claim of urban conflict is the basis of design creativity.
\end{abstract}

\section{Keywords}

Urban Spaces, Cities, Design, Urban Transformation, New Urbanism

\begin{abstract}
L'argomento è una riflessione sulla deriva dello spazio pubblico nelle città mediterranee, delle nuove tendenze che vanno dall'Urbanismo Tattico alle operazioni urbane che ripensano di nuovo il carattere pubblico delle nostre città.

La città è un organismo complesso che non può semplificarsi a volontà, ha la sua inerzia, il suo tempo e la sua difficoltà per digerire le ideologie politiche.

La rivendicazione del conflitto urbano come base della creatività progettuale.
\end{abstract}

\section{Parole chiave}

Spazio pubblico, Città, Progetto, Trasformazione Urbana, Nuovo Urbanismo 
La città cambia nello stesso modo in cui lo fanno anche le persone. Le vecchie città stanno lottando per la loro sopravvivenza, per il loro futuro, per essere più vivibili, più respirabili, più vicine alle persone che le abitano e, negli ultimi tempi, registrano sempre più evidenti metamorfosi che rompono schemi, distruggono previsioni e modificano obiettivi pianificati.

In un mondo in cui si presume che nei prossimi cinquant'anni oltre l' $80 \%$ della popolazione vivrà in ambienti urbani', la lotta per rettificare le tendenze e per conquistare la città per il cittadino è cruciale.

$\grave{E}$ in questo scenario che lıevoluzione della attuale pandemia colpisce una nuova crisi urbana in risposta ad una vera e propria crisi nel rapporto tra le persone, che si presenta particolarmente aggravata nei paesi del sud Europa, ovvero laddove ancor più questo rapporto si basa sullıaffetto, sul contatto e su ciò che si può chiamare calore umano.

Tutto questo ha un scenario ideale che è lo spazio pubblico, l' 'agorà', il luogo in cui si cristallizza la vita della città e dei suoi abitanti, in cui le persone interagiscono attraverso ogni tipo di immaginabili forme di associazioni vitali, come giochi, scambi commerciali, una passeggiata condivisa, rapporti personali di coppia o di gruppo, dipendenze legate a nuovi tipi di mobilità urbana, raggruppamenti per eventi, siano essi sociali o religiosi, o pura osservazione, d'es- sere parte di tutto questo circo umano trasformato in un spettacolo meraviglioso.

In questi tempi nuovi in cui viviamo, in cui è praticamente vietata, de facto, la staticità delle persone nello spazio libero, inibendo la possibilità di tessere relazioni, conversazioni o altre forme di socialità, lo spazio aperto si trasforma in un mero corridoio per nuove forme di spostamento nel quale non c'è nessuna chance per il gioco, lo scambio o gli affetti. Possiamo solo interpretare la città e il suo nuovo spazio pubblico come un susseguirsi di vicoli, di strade urbane con usi diversi dove cerchiamo di sviluppare e controllare tutte le nostre attività con la triste e ingenua volontà che non si verifichino conflitti tra di loro. A volte ci ossessionamo con l'applicazione della normativa, quando sappiamo tutti che la vera essenza e l'interesse della città si basano sul caso, l'indecisione e, in ultima analisi, la conflittualità. La città è "un milione di cose" si legge nel titolo di un vecchio programma radiofonico di Luís Arribas Castro emesso da Radio Barcelona, durante la decade degli anni '60, intuendo questa amalgama di situazioni e relazioni nella città, alcune delle quali appartenenti al mondo impercettibile dell'intangibile.

È in questi momenti di incertezza che si sceglie di lasciare quelle città che ci hanno visto crescere per cercare uno stile di vita più rurale, più umano, ipoteticamente più sano e indubbiamente più economi- 


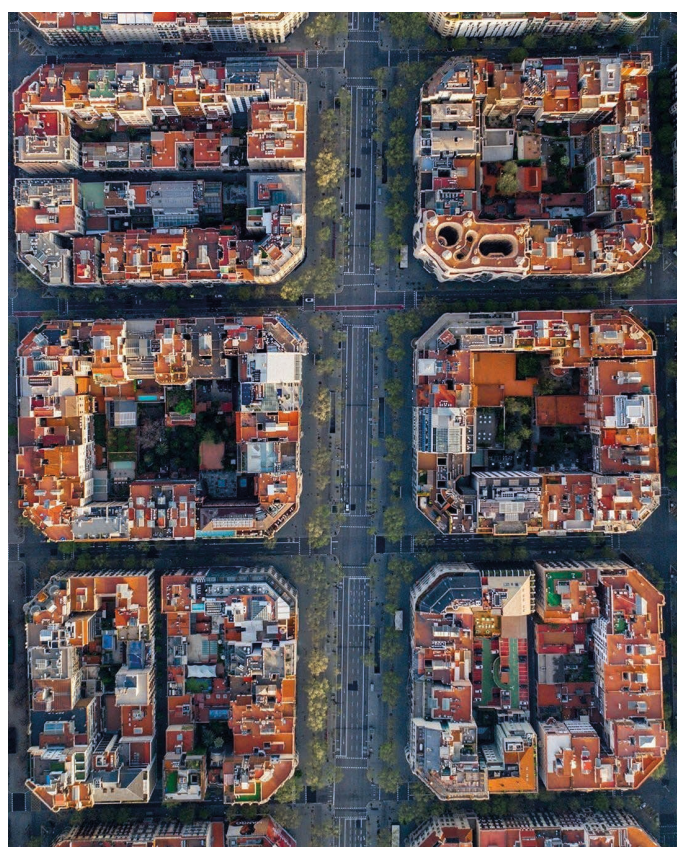

Fig. 1 - Barcellona vuota durante la pandèmia COVID19 Aprile 2020

co, con grande rassegnazione sociale, cercando un isolamento che ci salvaguardi dai diversi tipi di contaminazione o infezione che ci minacciano. II telelavoro facilita lıassunzione di queste decisioni, sebbene in molti casi significhi meno efficienza, il rifiuto di sane interferenze e un rapporto molto più incerto allsinterno del lavoro, poiché lıisolamento lo consente e lo facilita. Allo stesso tempo abbiamo iniziato ad essere espulsi dalla logica urbana dellseconomia derivata da una politica turistica esasperante, anche se paradossalmente in questi tempi di pandemia i turisti non esistono. Nel disperato tentativo di renderla più vivibile per il cittadino, la città è difatti sclerotizzata, meno efficiente e, ormai soffocata da affitti e acquisti pensati per livelli sociali altissimi, da terribili giochi di investimento di capitali stranieri o semplicemente dal più puro sradicamento sociale, tende ad espellere i suoi abitanti verso le periferie urbane.

La città occidentale contemporanea è ferma a un bivio, in una impasse di difficile prognosi futura e di no quando non sono vissuti, visitati, non riconosciuti e non utilizzati e il loro spazio pubblico perde tutto il suo significato sociale e quindi la sua essenza. Piazze senza bambini o anziani, parchi in attesa di essere salvati dalla loro chiusura e strade tristi, prevalentemente senza commercio e quindi senza movimento e senza quell'appeal sociale dalla luce suggestiva che si trova nelle vetrine o nei messaggi pubblicitari.

E al contrario di tutto questo, alcuni appartamen$\mathrm{ti}$, alcune case che sono nettamente presenti per le persone che le abitano, in misura maggiore di quanto non lo fossero mai state, si mostrano in tutta la loro realtà spesso triste e martellante, in cui le persone cercano la sotto privatizzazione dei propri spazi aperti inserendo una sedia, o occupando un angolo, che le fa isolare nell'isolamento, recuperando i loro micro paesaggi più quotidiani e vicini, mentre allo stesso tempo possono essere rilevati e riscoperti dalla finestra, nel migliore dei casi, i suoi orizzonti più lontani. Torniamo con un colpo ad essere più consapevoli della crisi di classe, della crisi sociale che non ci ha mai abbandonati.

Il paesaggio urbano, l'agognato spazio pubblico ci ha resi davvero più uguali, più sociali e quindi più persone. Stiamo perdendo, drasticamente, i nostri paesaggi intermedi, i paesaggi del nostro discorso vitale, quelli dell'interazione, della vita quotidiana, in cui ci siamo sentiti molto a nostro agio, i paesaggi della relazione, del viaggio, i paesaggi mutevoli di ciò che è abituale: tutte scene di una vita che è stata drasticamente ridotta. II tempo ci dirà se sarà tutto un esercizio da cui imparare o solo un brutto sogno senza fine. In questa delicata situazione, le nostre città non possono essere sottoposte da opportunisti guru teorici o politici a una riduzione dell'analisi delle virtù e dei problemi inerenti il futuro dello spazio pubblico. Quanto più vicini alla demagogia ci troviamo situati, quanto tutto sembra più comodo e semplice. Ma le questioni urbane, oltre ad essere complesse e spesso intangibili, non possono essere interpreta- 


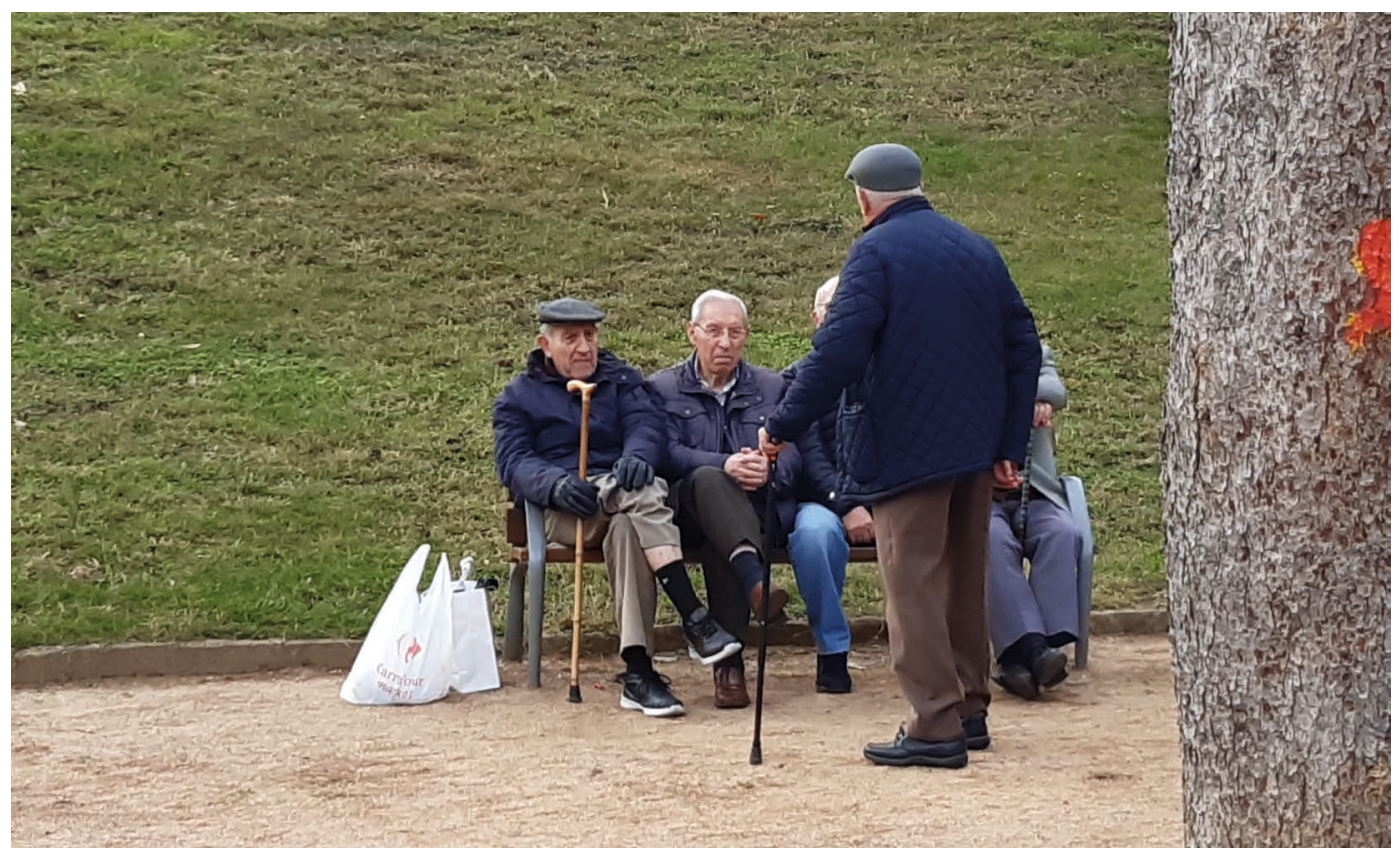

Fig. 2 - Spazio pubblico classico interattivo.

te dal punto di vista del più immobile reazionismo seguendo una pura ideologia, o più semplicemente improvvisando o andando verso un modello futuro che in nessun modo si puó considerare accettabile. La città si evolve e il suo ritmo diventa paragonabile allo spostamento di un grande transatlantico, con movimenti lenti, precisi e influenzati dal grande condizionamento che è la sua naturale e conosciuta inerzia. Le decisioni affrettate in città possono portare a risultati imprevedibili, molti dei quali sicuramente disastrosi.

La pianificazione urbana richiede tempo, sensibilità e sfumature.

La metropoli attuale e futura si caratterizza per i suoi grandi problemi di mobilità, basati sulla difficoltà di cambiare paradigma. Dall'attuale base di città supportata in larga misura dalla mobilità circolatoria dell'auto privata, deve però oscillare gradualmente, ma costantemente, verso il predominio del trasporto pubblico, ovvero verso il cosiddetto slow motion, in cui il pedone diviene il vero cittadino del futuro.
Tutto ciò porterà ad una conurbazione più pulita e sana, visto che l'attuale situazione dell'emissione di gas nel mondo non può attendere né ritardi, né dubbi per la sua drastica riduzione. Con questo, sempre più enfasi viene posta sui vantaggi di vivere la città a piedi, e di questo abbiamo una letteratura infinita che va dalla 'peripatetica' ellenica, attraverso Rousseau, Stevenson, Baudelaire, Sebald, Walser o Thoreau, alla fragorosa rivoluzione del camminare o del passeggiare come filosofia umana o medicina per la mente e il corpo, anche al camminare inteso come un atto sovversivo spontaneo.

A sottolineare queste riflessioni si citano ad esempio tra i tanti le ultime opere ed esperienze di Francesco Careri, sulla scia dei situazionisti, in Walkscapes, il contributo di poeti, scrittori, artisti e scienziati di ultima generazione in opere come Wonderlust. A history of walking di Rebecca Solnit, o Éloge de la marche di David Le Breton, Walking di Erling Kagge, il lavoro scientifico divertente e applicabile di Shane 


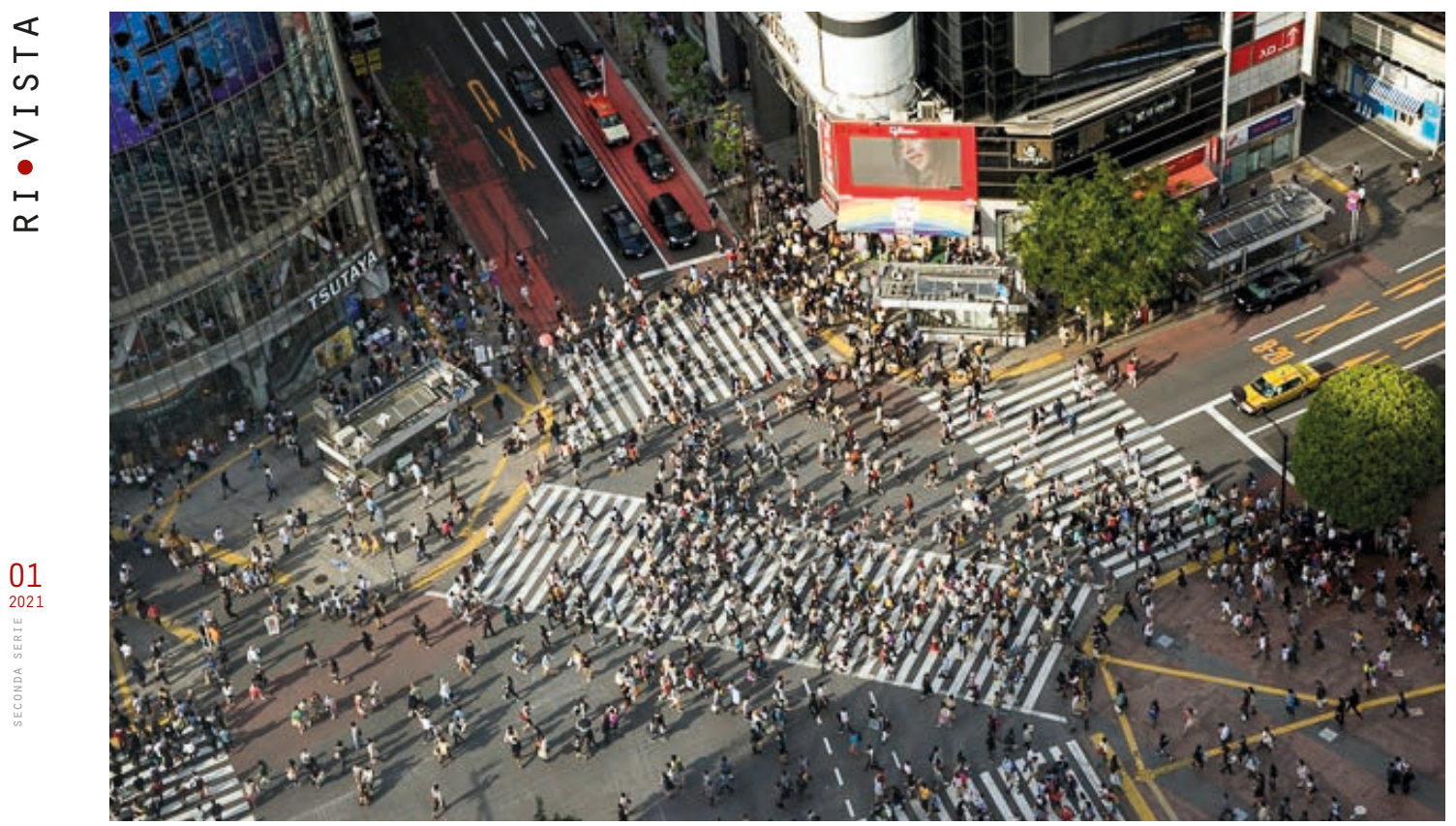

Fig. 3 - Incrocio in Shibuya, Tokio.

O'Mara In Praise of Walking o la dolce esperienza del norvegese Torbjorn Ekelund in Stiens historie.

In alcuni casi sorprendono studi come La ciudad paseable del Ministero dello Sviluppo spagnolo e del Ministero dell'Ambiente (Echavarri, Daudé, Porto Schettino, 2009), in cui i pedoni sono gli utenti di riferimento dei piani urbani comunali, dei piani parziali o, anche più in dettaglio, direttamente dell'attività di progetto.

Non vanno dimenticati altri mezzi di trasporto non inquinanti nell'ambiente urbano. Tra tutti spicca l'uso della bicicletta, di non facile attuazione, soprattutto nelle città con una certa topografia così come nei centri storici, che per logica storica sono privi di misure e distanze adeguate, o a causa della necessità quasi banale che i percorsi basati sulla bicicletta siano circuiti eminentemente chiusi e organizzati in reti. Sulle biciclette ci sono tante opinioni, scritti, associazioni e appassionati, e tra questi vorrei evidenziare un paio di libri, riferimenti personali, che trovo particolarmente 144 suggestivi e attuali: Éloge de la bicyclette dell'antro- pologo francese Marc Augé, autore tra tante opere di Non-lieux. Introduction à une anthropologie de la surmodernité, e la squisita opera Bicycle Diaries di David Byrne, musicista scozzese, ex leader dei Talking Heads, che ci racconta in modo delizioso le tue visite in bicicletta nelle principali capitali del mondo.

Pedoni statici o in movimento lineare, biciclette, altri mezzi di trasporto individuali non inquinanti, trasporti pubblici (autobus, tram e taxi) e veicoli privati devono coesistere in uno spazio predeterminato e in molte occasioni molto scarso, questo è dovuto alla necessaria revisione dello spazio pubblico della città ereditato dal XIX secolo, nel migliore dei casi.

Ma questa esigenza di sicurezza, dal difficile risultato formale, in cui ogni uso deve essere assorbito nello spazio contingente, ha una componente pericolosa che separa rigidamente, individualizza e normalizza quella che a torto è l'essenza dello spirito urbano: la loro interconnessione, ibridazione e libertà. In tutto questo, e scommettendo su una città amabile in cui non si verifichino interferenze pericolose o 


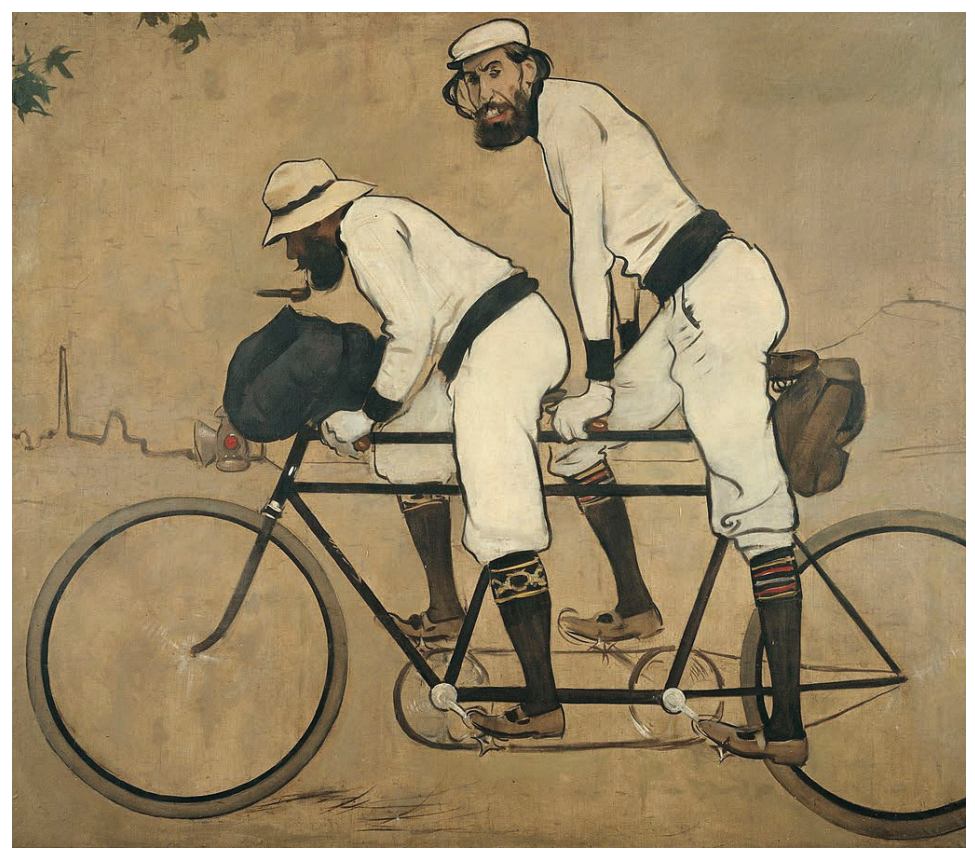

Fig. 4 - Elogio della bicicleta (Tandem. Ramon Casas).

frizioni vitali drammatiche se conviene ricordare la differenza della sfumatura, quella della suggestiva conflittualità come ricchezza di convivenza in una città, sono il suddetto contatto e la trasversalità sociale gli elementi che generano progresso urbano. In questa domanda si evidenzia il lavoro e l'esperienza dell'ingegnere olandese Hans Monderman (1945-2008), come innovatore dello spazio stradale urbano in cui ha ideato la proposta di 'spazio condiviso', dove vengono eliminate le divisioni reali tra traffico veicolare e per i pedoni, con l'eliminazione degli elementi che caratterizzano queste separazioni quali cordoli, segnaletica, semafori, regolamenti, ecc ... (Monderman, 2007).

Monderman ha insistito sul fatto che l'efficienza del funzionamento della strada viene migliorata con il suo nuovo design stradale con la proposta attraente che ogni utente dello spazio pubblico negozi i suoi usi direttamente con gli altri attori.

Una delle proposte che aggiunse per costruire la sua ipotesi fu il progetto che chiamò Woonerf o Living
Street da un'iniziativa popolare vissuta a Delft nel 1968 e che lo portò alla progettazione di oltre 6.000 strade. Quasi sempre, ovviamente, nelle aree di sviluppo urbano e di riqualificazione olandese.

Nell'ambito della città centrale, è degna di nota l'operazione londinese di Exhibition Road a South Kensington, proposta fatta per uno spazio condiviso, ispirata all'opera classica di Urbanism Traffic in towns di Colin Buchanan (Buchanan, 1963). Questa operazione urbana progettata nel 2003 da Dixon e Jones, su un progetto per l'Esposizione Universale del 1851, e completata per i Giochi Olimpici di Londra nel 2012, è uno dei notevoli esempi di una soluzione urbana basata sull'educazione e la tolleranza, non nella regolamentazione, nella segregazione e nella sicurezza falsa e fuorviante. Exhibition Road è una strada che non ammette alcun tipo di corsia urbana, né separazione dei suoi diversi usi. Una vera 'strada condivisa'.

Questi esempi citati coincidono con gli obiettivi della necessità di effettuare un cambiamento paradig- 


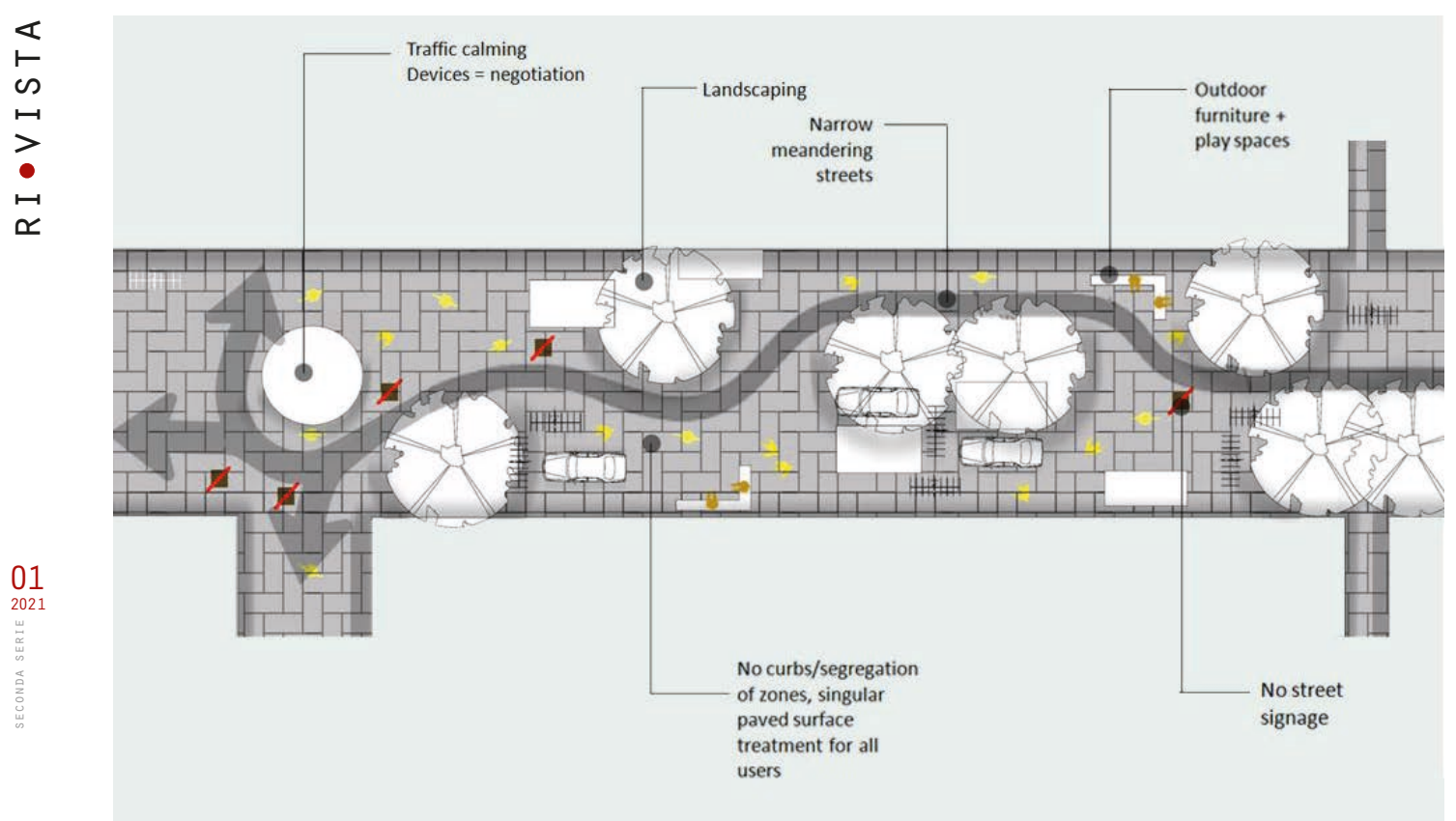

Fig. 5 -Progetto di Woonerf (M. Monderman).

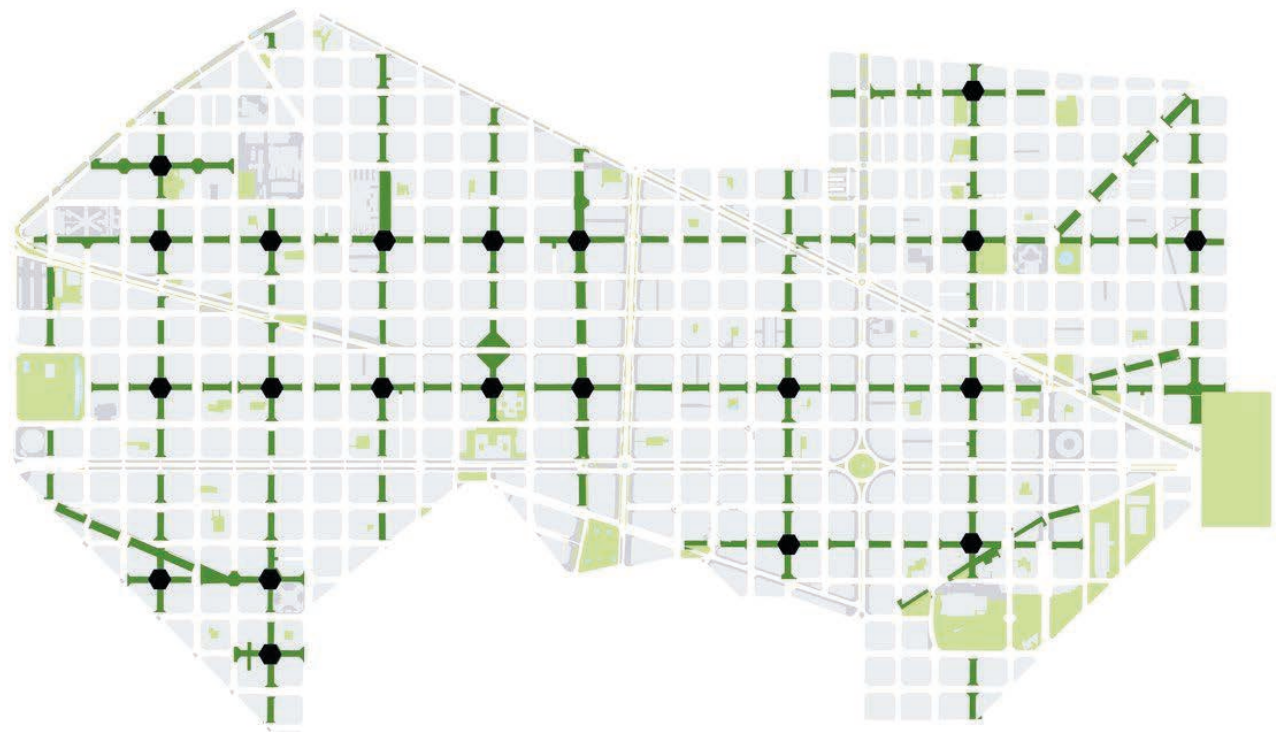

Fig. 6 - Nuovi assi e piazze di Barcellona. 2020.

pagina a fronte

Fig. 7 - Exhibition Road. South Kensington, Londra. 

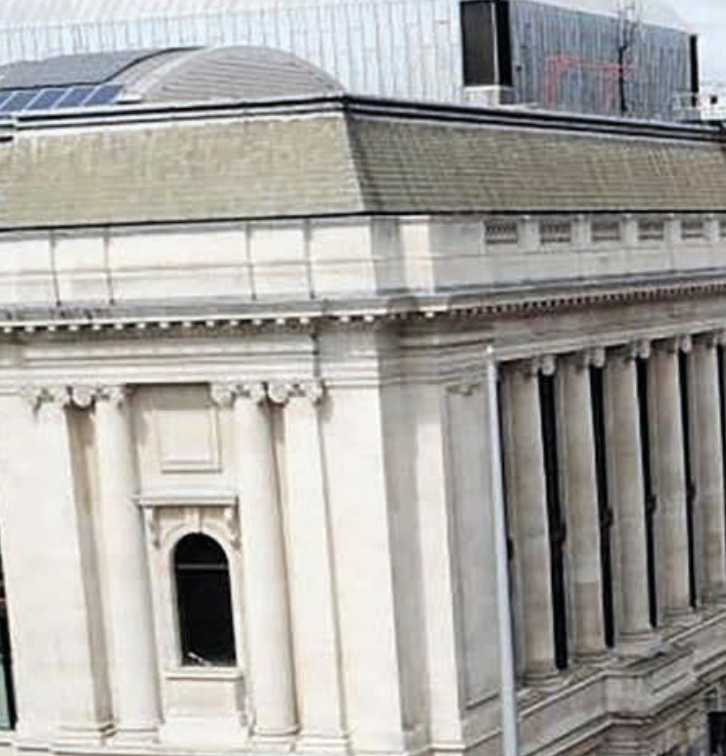

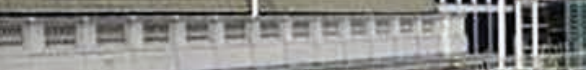

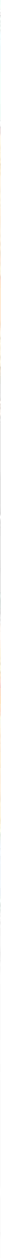




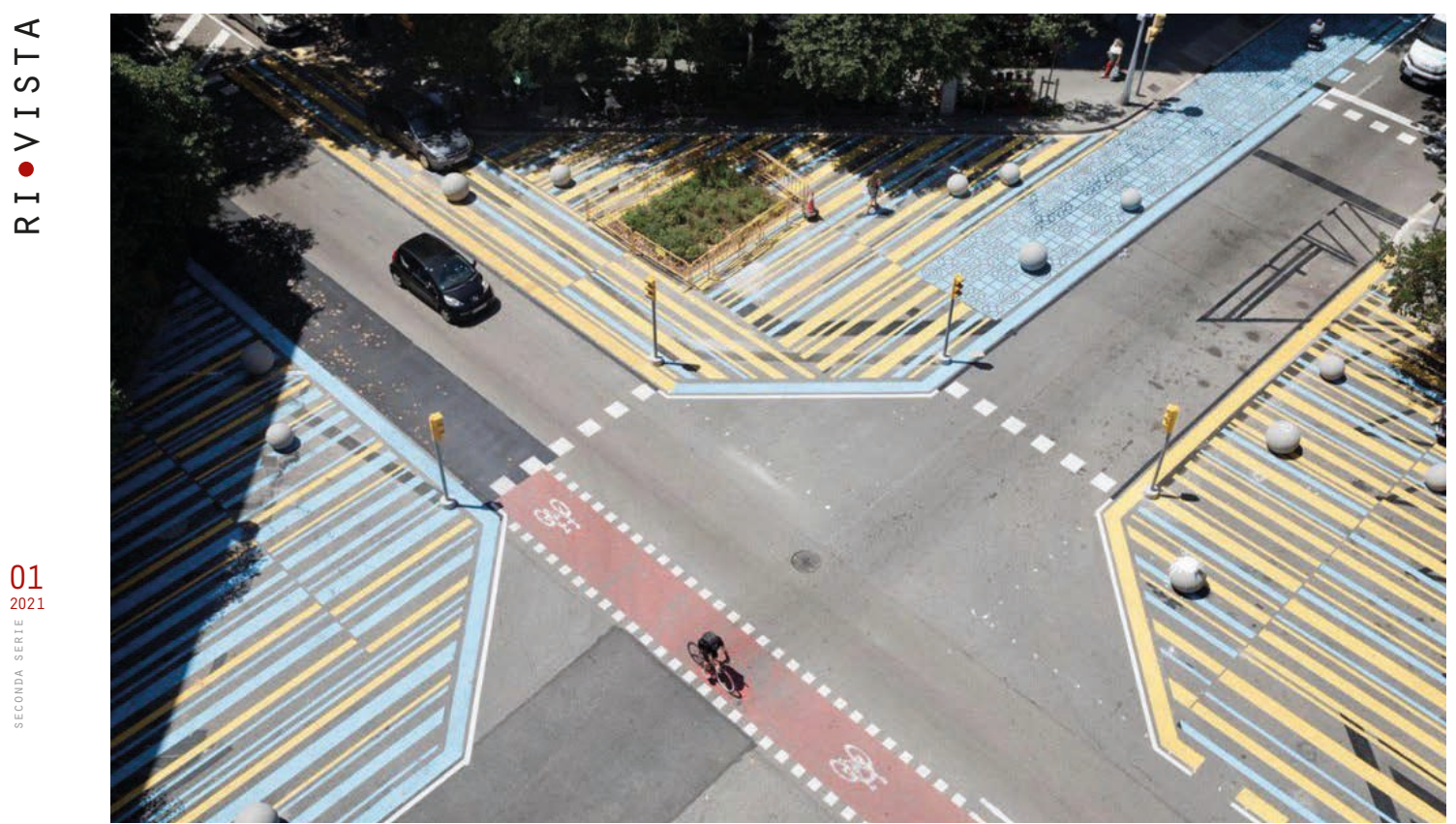

Fig. 8 - Urbanismo tactico

matico nello spazio pubblico urbano, ricercando una soluzione condivisa e serena tra i diversi utenti del luogo e non necessariamente e prematuramente adottando la logica della legge e conseguentemente della normativa che in tante occasioni agisce volendo 'concordare' tra meccanismi e forme differenziate, anche antagoniste, in proposte di grande difficoltà formale, cognitiva ed estetica.

Nella città di Barcellona, in questo momento, sono in corso un numero significativo di iniziative comunali, approfittando della decompressione della situazione urbana in cui i cittadini, i visitatori e con loro il traffico sono notevolmente diminuiti, sostenuti dal mal definito Urbanismo tattico, che si diffonde precariamente per strade, viali e piazze, in attesa di avere le risorse per renderla definitiva e che il dibattito urbano ne fornisca le ragioni.

Barcellona si propone nel suo centro vitale come un insieme di superblocchi, una rete di assi verdi e un insieme di piazze rivisitate al suo crocevia. Soluzio- zione della città, la renderanno più diseguale se possibile e in termini generali ne determineranno una inarrestabile gentrificazione, provocando un effetto inverso a quanto si desidera ottenere. Un impareggiabile parco tematico urbano per i visitatori che lo popoleranno quando possibile.

Quello che inizia a essere conosciuto come il Superblock Barcelona, una città che negli anni Ottanta del secolo scorso ha rivoluzionato la strategia del design dello spazio pubblico e che ha trasformato le sue strade e piazze in un vero laboratorio contemporaneo sullo spazio pubblico, ispirazione per tante altre città negli ultimi tempi, non può commettere un grosso errore con gravi conseguenze per la città a causa della necessità imperativa di risultati elettorali o di ottiche che non eccedono la visione di quartiere.

È importante riconoscere le diverse situazioni urbane, non solo per i loro problemi intrinseci ma anche per le loro reali esigenze e possibilità. La città non è una pagina bianca, hanno sottolineato Pep Parceri- 


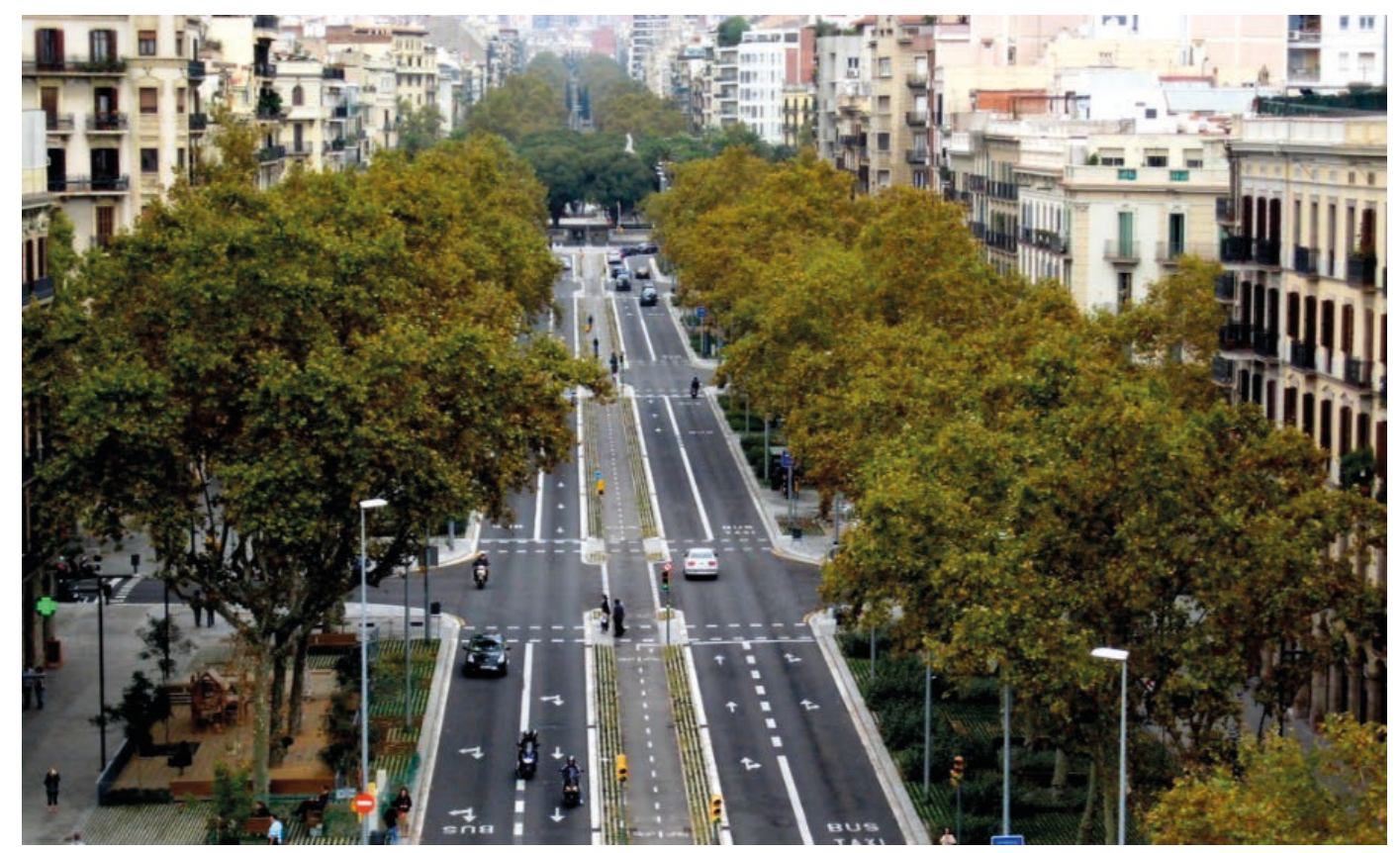

Fig. 9 - La vegetazione della città. Passeig de Sant Joan, Barcellona.

sa e María Rubert de Ventós, professori di Urbanistica a Barcellona, in una raccolta urbana del campo dell'insegnamento (Parcerisa Bundó, Ventós 2014), ma con un percorso e una portata più ampio, interpretando il campo urbano come una giustapposizione di strati di informazioni storiche, usi, volontà e speranze.

Una volontà tecnica, politica, ideologica o anche propagandistica non può imporsi su una realtà complessa, ricca, intelligente e sedimentata come le nostre città.

II paesaggismo ci ha insegnato tanto, e non solo a proporre la rivegetazione delle città come semplice risorsa per la sua nuova immagine, sotto lo slogan del 'disasfaltare' la città. II paesaggio ben compreso, e ciò che difendiamo, è quello che considera il riconoscimento nel territorio di tanti strati di informazioni come corsi d'acqua, habitat, connettori, geologia, usi, atmosfere, leggi ecologiche o gradi di protezione dei luoghi, tra le altre cose. La città deve essere trattata esattamente come il paesaggio, con un'azione sensibile a tutti i livelli di informazione che ci fornisce.

L'architetto e comunicatore Xavier Monteys si espresse in modo lucido e con lungimiranza quando nel suo delizioso libro // piacere della città (Monteys Roig, 2013), pieno di prelibatezze intellettuali scritte da fruttuose passeggiate alla moda del flâneur contemporaneo, disse: "La città diventa il contrasto, nell'elemento contro cui giudichiamo, nella grande scena dell'attività, tra l'altro, perché la città è il predominio dell'uso delle cose. La sola vista di una città vuota si rivela, dopo pochi istanti, se dura, un'idea e un luogo terrificanti. La città è la gente, la gente che la spende, anche solo guardandola. In questo senso mi piace la definizione di spazio pubblico - strada o piazza - proposta dall'architetto Jan Gehl quando afferma che è "vita sociale tra edifici”. Questo 'tra' contiene tutto, inclusa l'architettura". 2

È assolutamente determinante riconoscere la metropoli non come un sito, una somma di spazi pubblici, dove nuove proposte possono essere imple- 


\section{Note}

mentate da zero: la città è interattività, è storia, è ${ }^{1}$ Vedi United Nations, Revision of World Population Prospects, https://population.un.org/wpp/ ${ }^{2}$ Traduzione a cura dell'autore Tratto dal libro di atmosfera ed è un accumulo di informazioni, che in alcuni casi sono addirittura in contraddittorio. QueMonteys X. 2012, El Plaer de la Ciutat, Girona, Ed. EPS sta è la città.

Universitat de Girona, p. 13.

Allo stesso tempo, il richiamo incisivo che l'architetto e urbanista Manuel de Solà-Morales ci lascia in Ciutats, cantonades quando cita Aristotele dicendo "una città è composta da diversi tipi di uomini; persone simili non possono fare una città".

La città non è l'individualità. Nella città predomina ciò che è relazionale, vediamo gli elementi, ma li vediamo, allo stesso tempo, uno in relazione all'altro, accanto o davanti, permettendo alla memoria di collegare le esperienze.

Lo spazio pubblico urbano è rapporto umano e il rapporto tra le persone sarà ancora una volta la base $\mathrm{e}$ la ragione della città futura. 


\section{Bibliografia e sitografia}

Buchanan C. 1963, Traffic in Towns. A study of the long term problems of traffic in urban areas, H. M. Stationery Office, London.

Echavarri J.P., Daudén F.J.L., Porto Schettino M. 2009, La ciudad paseable Recomendaciones para la consideración de los peatones en el planeamiento, el diseño urbano y la arquitectura Cobierno de España - CEDEX, Madrid.

Gehl, J. 2010, Ciudades para la gente, Ediciones Infinito, Buenos Aires.

Monderman H. 2007, Rethinking the design of streets and public space, Urban Design London, disponibile in http://www.urbannous.org.uk/udlhm1.htm (12/2020).

Monteys Roig, X. 2013, El plaer de la ciutat - Taking pleasure in our cities, Hilando Libros, Madrid.

Parcerisa Bundó J., Ventós de M. R. 2014, La ciudad no es una hoja en blanco, Laboratori d'Urbanisme de Barcelona, Barcelona.

Solà-Morales, de M. 2004, Ciutats Cantonades. Catalogo della Mostra Ciutats Cantonades, Forum 2004, Barcellona.

Solà-Morales, de M. 2021, Miradas sobre la ciudad, Acantilado, Barcellona.

United Nations, Revision of World Population Prospects, https://population.un.org/wpp/ (12/2020) 